\title{
Measures of the components of the Galactic diffuse emission with INTEGRAL/SPI
}

\section{Bouchet*, E. Jourdain, J.-P. Roques}

Université de Toulouse, UPS-OMP, IRAP, Toulouse, France

CNRS, IRAP, 9 Av. colonel Roche, BP 44346, F-31028 Toulouse cedex 4, France

E-mail: liboucheteirap.omp.eu

\section{A. W. Strong}

Max-Planck-Institut für extraterrestrische Physik, Postfach 1603, 85740 Garching, Germany

The hard X-ray/soft gamma-ray emission of the Galaxy consists of several components, linked to specific physical processes or sources populations. We use the SPI spectrometer aboard the INTEGRAL observatory to disentangle the spatial morphology and the spectral shape of these various contributions. Based on 6 years of data, we have measured a detailed ridge spectrum and constrained the spatial distribution of the emission from $20 \mathrm{keV}$ to $2.5 \mathrm{MeV}$. The diffuse continuum spectrum and its spatial distribution are in relatively good agreement with predictions from GALPROP models. In a second complementary step, the flux of the $511 \mathrm{keV}$ positron annihilation line, the ortho-positronium continuum, the ${ }^{26} \mathrm{Al}$ and the ${ }^{60} \mathrm{Fe}$ lines have been measured with improved precision. Their spatial distributions as well as other characteristics (positronium fraction, ${ }^{26} \mathrm{Al} /{ }^{60} \mathrm{Fe}$ ratio,...) have been estimated and compared with theoretical expectations.

An INTEGRAL view of the high-energy sky (the first 10 years) - 9th INTEGRAL Workshop and celebration of the 10th anniversary of the launch

15-19 October 2012

Bibliotheque Nationale de France, Paris, France

\footnotetext{
* Speaker.
} 


\section{Introduction}

The hard X-ray/soft gamma-ray emission of our Galaxy consists of several components linked to specific physical processes or objects. The spectrometer SPI [1], 2] has the capacity to image both point sources and extended/diffuse emission components over a large field of view of $30^{\circ}$. The instrument is suited to disentangle the spatial morphology and the spectral shape of these various contributions. Public data recorded between February 2003 and January 2009 representing $\sim 1.1$ $\times 10^{8} \mathrm{~s}$ observation livetime are used for this purpose. In our previous work ([3] and references therein) we have presented sky maps and spectra of the Galactic diffuse continuum components and demonstrated the presence of a hard power-law continuum emission, which was interpreted as inverse-Compton emission from cosmic-ray (CR) electrons and positrons upscattering the Galactic interstellar radiation field (ISRF). In the present analysis, we also study the annihilation radiation spectrum (511 keV line and ortho-positronium continuum), and ${ }^{26} \mathrm{Al}$ and ${ }^{60} \mathrm{Fe}$ radioactive lines, which represent two other important large-scale components in the Galactic emission. So far to date, only COMPTEL has mapped the ${ }^{26} \mathrm{Al}$ during its 9 years survey ([4] and references therein). $S P I$ gives us the second opportunity to get some pieces of information about its spatial distribution.

\section{Point-sources emission}

Point-sources contribution is extracted from the data, simultaneously with the diffuse and background components, hence a census of sources is also derived during the analysis. Thanks to dedicated data segmentation code [ $[$ ], this data reduction process takes into account the intensity variations of the sources. The resulting SPI all-sky survey allows us to detect $\sim 280$ point sources. The average emission spectrum of each of these sources can be viewed at http://sigma-2/integral. Figure 1 displays the different contributions to the Galactic emission spectrum between 20 and $2500 \mathrm{keV}$. We see that the point-sources emission explained $\sim 90 \%$ of the total emission around 50 $\mathrm{keV}$.

\section{Galactic diffuse continuum emission}

One of the main objectives was to compare the SPI measurements of the diffuse continuum emission to the prediction of a CR propagation code. To predict Galactic diffuse emission in the energy range from $\mathrm{keV}$ to $\mathrm{TeV}$ energies, we use the GALPROP code including a new model for the Galactic interstellar radiation field (ISRF) [6].The reference model (GALPROP ID 54_z04LMS) is based on the electron (plus positron) spectrum measured by Fermi-LAT [7]). It has a halo height of $4 \mathrm{kpc}$ and includes CR re-acceleration; for further details see [8]. The measured distribution of the emission in longitude and latitude over the whole Galaxy was compared to template maps. Many combinations of templates were tested, the detailed procedure is described in [3]. The best fit template map consists of a combination of GALPROP Inverse-Compton (IC) distribution, $4.9 \mu$ map. The modeling and the study of the radioactives lines and annihilation radiation spectrum are detailed in sec. $\$$ and 5 . Finally, we have been able to isolate each of the "diffuse" components. Figure 1 shows the diffuse emission intensity in the central radian $\left(|l|<30^{\circ},|b|<15^{\circ}\right)$. The diffuse emission has the following main features: 


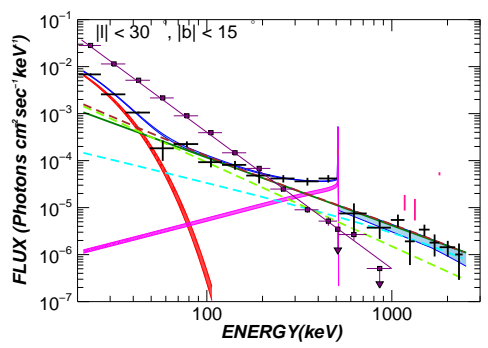

Figure 1: Spectra of the different emission components in the central radian of the Galaxy $\left(|l|<30^{\circ}\right.$ and $\left.|b|<15^{\circ}\right)$. SPI measurements are black crosses. Violet squares: total emission of resolved sources. Violet line: power law fit to the resolved sources emission (power law index is 2.9 and flux at $100 \mathrm{keV}$ is $4 \times 10^{-4} \mathrm{ph} \mathrm{cm}^{-2} \mathrm{~s}^{-1} \mathrm{keV}^{-1}$ ). Blue: total diffuse emission - Magenta: annihilation radiation spectrum (line + positronium). Red: Emission of low energy "unresolved" sources. The possible ranges of variation of these components are represented with shaded areas. Dark green line - is the continuum emission thought to be dominated by interstellar particle interactions. The diffuse continuum best fit spectrum based both on spatial morphology and spectral decomposition is indicated by the dashed cyan $(4.9 \mu$ spatial component $)$ and green dashed (IC component) lines. The sum of these two components is the brown dashed line which is very similar to the power law fit of index 1.44 based solely on spectral decomposition (dark green line).

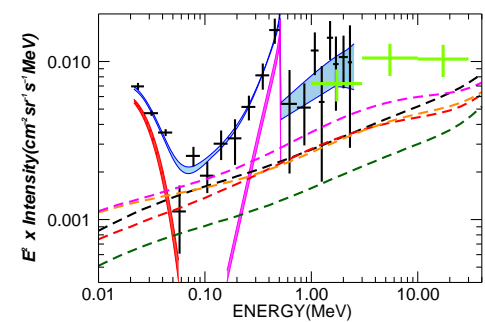

Figure 2: Same as figure 1 with different GALPROP configurations. The dashed dotted lines are IC models. Primary electron spectrum based on FermiLAT (dark green), primary electron spectrum measured by Fermi-LAT increased by a factor 2 (black), increased halo height from $4 \mathrm{kpc}$ to $10 \mathrm{kpc}$ (red) and increased ISRF in the Galactic bulge (x 10) (orange) and both increased halo height from $4 \mathrm{kpc}$ to $10 \mathrm{kpc}$ and ISRF in the Galactic bulge (x 10) (magenta).

- The IC emission distribution predicted by the GALPROP code is in fair agreement with the data (fig. 2). However, a model with an electron spectrum increased by a factor 2 over the standard model based on the electrons (plus positrons) measured by Fermi-LAT is in better agreement. Also, an increased ISRF in the Galactic bulge or a large Galactic CR halo are other reasonable possibilities to that can lead to an increased flux. The data analyzed in this paper do not allow to discriminate between these possibilities.

- An additional component is required below $50 \mathrm{keV}$. This excess over the IC emission is well modeled with the NIR/DIRBE $4.9 \mu \mathrm{m}$ map. This low-energy component has an exponential spectrum with a cutoff at $8 \mathrm{keV}$ and a flux at $50 \mathrm{keV}$ of $\sim 2 \times 10^{-4} \mathrm{ph} \mathrm{cm}^{-2} \mathrm{~s}^{-1} \mathrm{keV}^{-1}$ in the central radian. It can interpreted in terms of the stellar origin(CVs population) as proposed by [9].

The diffuse continuum emission spectrum obtained with the present analysis confirms and improves the results reported in [10] and [6]. Below $100 \mathrm{keV}$, it is also compatible with results obtained from INTEGRAL/IBIS analysis [9, 11]. In addition,

- The diffuse continuum flux around $1 \mathrm{MeV}$ presents a good agreement with the COMPTEL/CGRO measurement.

- There is no detection in the SPI energy range of the "Fermi bubbles" at the present level of sensitivity.

\section{Radioactive ${ }^{26} \mathrm{Al}$ and ${ }^{60} \mathrm{Fe}$ lines}

The ${ }^{26} \mathrm{Al}$ emission has been already studied with the SPI instrument ([12] and references therein) and appears, with the present analysis, as a $\sim 15 \sigma$ detection with a flux around $2.7 \times 10^{-4} \mathrm{ph}$ 
$\mathrm{cm}^{-2} \mathrm{~s}^{-1}$. However, only the COMPTEL instrument has studied the emission distribution of the ${ }^{26} A l$ over the whole sky. To start such study with $S P I$ we model its emission distribution with several template maps with various latitude and longitude extensions, as well as different contrasts (defined as the ratio of the flux enclosed in the region $|l|<150^{\circ},|b|<15^{\circ}$ to the total flux), in order to determine the most appropriate one. The main characteristics of these templates and the data analysis methodology are detailed in [16].

Due to its weakness, the ${ }^{60} \mathrm{Fe}$ line emission has been detected clearly from our Galaxy with only two instruments, RHESSI [13] and $S P I\left[14\right.$, 15], with a deduced ${ }^{60} \mathrm{Fe} /{ }^{26} \mathrm{Al}$ ratio of $\sim 0.15$. It is not possible to derive constraints on the spatial distribution of the ${ }^{60} \mathrm{Fe}$ isotope (1173.23 and $1332.50 \mathrm{keV}$ ) lines due to their weak fluxes. The ${ }^{60} \mathrm{Fe}$ and ${ }^{26} \mathrm{Al}$ radioactive lines emission come mainly from the Galactic plane, we assume in the present analysis that they follow the same spatial distribution. This is physically reasonable since ${ }^{26} \mathrm{Al}$ and ${ }^{60} \mathrm{Fe}$ are believed to share at least some of their production sites, (e. g., massive stars and supernovae ([21, 22]).

The ${ }^{26} \mathrm{Al}$ emission is studied in 1806-1812 keV energy band and the two isotopes of the ${ }^{60} \mathrm{Fe}$ line in the 1170-1176 and 1330-1336 keV bands. At these energies, the instrumental background is the main contributor to the count rate recorded by SPI. Thus, the background modeling is a key issue, since the lines signal-to-noise ratio are below $1 \%$. However, the present analysis of these lines is an "imaging" analysis and does not require a sophisticated instrumental background modeling. It does not use the bands adjacent to the lines to establish a database for the background modeling [12]. It simply relies on the ability of SPI to discern the signal of the background and the signal of sources, thanks to the coded-mask imaging system.

\subsection{Background modeling}

The background detector pattern (relative detector count rates or uniformity map) has been shown to be stable on $\sim 6$ months timescale, between two successive annealings [1], with a global intensity varying on a shorter timescale of $\sim 6$ hours. The patterns (one per six months) can be either hand fixed before the fitting procedure ("empty-field" templates) or computed during the data reduction process [17, 3]). To determine a priori the patterns, as the dedicated SPI "emptyfield" observations are rare, the exposures whose pointing latitude satisfies $|b|>30^{\circ}$ constitute a good approximation. On the other hand, if the detector patterns are fitted simultaneously with the sky model (adjusted pattern method), they are model-dependent, the global sky model being more flexible. It is also possible to use a more complex background model by including an additional component, such as an additional isotropic term. However, such a contribution is difficult to assess (mathematical degeneracy) and does not allow to bring additional useful information. Consequently, it has not been further considered (See the discussion in [3]).

\subsection{Potential bias : Non-uniform sky exposure}

An important source of potential bias in the data comes from the sky exposure. It is very far from being uniform : most of the exposures are concentrated along the galactic plane and especially the central region of the Galaxy. The induced effects have been estimated using simulated data for each tested sky map. From that, we have concluded that recovered fluxes depend on the assumed spatial morphology and the background model. Using simulations, we found that it could be overestimated up to $\sim 15 \%$ and $\sim 20 \%$ for the adjusted pattern and "empty-field" derived pattern 


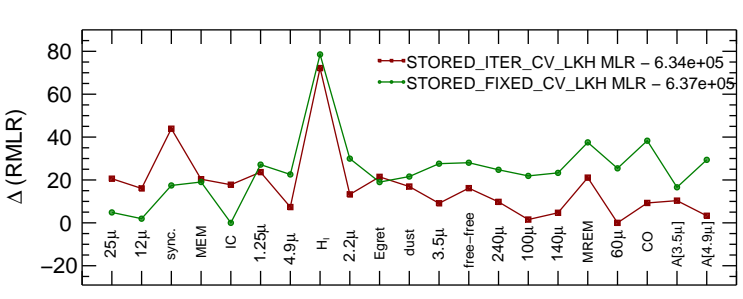

Figure 3: Terms "sync.", "dust" and "free-free", are abbreviations for $53 \mathrm{GHz}$ synchrotron, $53 \mathrm{GHz}$ dust and $53 \mathrm{GHz}$ free-free maps. MEM and RMEM indicate the COMPTEL maps $\|, \mathrm{A}[3.5 \mu]$ and $\mathrm{A}[4.9 \mu]$, the corrected NIR extinction maps. $25 \mu, 12 \mu$ are MIR maps, $1.25 \mu$ to $4.9 \mu$ are NIR maps and $60 \mu$ to $240 \mu$ FIR maps. IC is InverseCompton predicted map with the GALPROP code. The results are obtained with two different background pattern modelings: the first one, (background pattern adjusted), is shown in red and filled red square. the second one ("empty-field" derived pattern) is shown in green and filled red circle. The maps are ordered following their contrast defined as the ratio of the flux enclosed in the region $|l|<150^{\circ},|b|<15^{\circ}$ to the total flux. This ratio is 0.4 for $25 \mu$ map, $\sim 1$ for $\mathrm{A}[4.9 \mu]$ map and 0.7 for EGRET map (tracer of interstella gas(cosmic-ays). The maps up to EGRET (left part) a gas/cosmic-rays). The maps up to EGRET (left part) are ${ }^{\text {aid }}$ to have low-contrast and
above (right part) high-contrast. Details can be found in [16].

methods respectively, considering a 13-15 $\sigma$ detection, representative of the ${ }^{26} \mathrm{Al}$ line. However, correction factors can be applied to obtain an rough estimation of the ${ }^{26} \mathrm{Al}$ and ${ }^{60} \mathrm{Fe}$ fluxes.

\subsection{Results}

Figure 3 shows the Maximum Likelihood Ratios obtained the different tested maps. We display the results obtained with the different modelings of the instrumental background. We can first note some interesting points: while, in general, the "empty-field" derived pattern method tends to favor the low contrast maps, the opposite trend is observed with the the adjusted pattern method. Second, the above analysis helps to exclude some maps (53 GHz synchrotron which traces the cosmic-rays/magnetic field (sync.), Neutral hydrogen $\left(\mathrm{H}_{I}\right)$ and also to some extent the COMPTEL MREM image of the ${ }^{26} \mathrm{Al}$; a schematic representation of the emission observed by COMPTEL), but not to distinguish between the others. It is difficult to firmly conclude about a unique solution: What we could say is that in the "low-contrast" part (up to EGRET map), 12 or $25 \mu$ MIR and IC maps appear as the best tracers while for high contrast maps, the FIR 60 to $240 \mu$ and $53 \mathrm{GHz}$ free-free maps will be preferred. It should be noted that whatever the map templates used, all the measured fluxes are compatible with the value of $2.8 \times 10^{-4} \mathrm{ph} \mathrm{cm}^{-2} \mathrm{~s}^{-1}$ (excepted the $\mathrm{H}_{I}$ map).

We have also looked for some local excesses from regions identified as potential emitters of ${ }^{26} A l$, by adding to our sky model the Sco-Cen, Cygnus, Vela and Carina complexes. The considered spatial extensions are based on the recent SPI investigations ([18-20]) and we found compatible fluxes with these authors, even though the estimated fluxes depend to some extent on the sky model. For all the tested distributions, the ${ }^{60} \mathrm{Fe}$ isotope lines are detected at a level of $\sim 3 \sigma$ to $\sim 4 \sigma$, respectively in the $1170-1176$ and $1330-1336 \mathrm{keV}$ bands. Their global mean flux in the inner Galaxy is $\sim 5 \times 10^{-5} \mathrm{ph} \mathrm{cm}^{-2} \mathrm{~s}^{-1}$.

While poorly constrained, the ${ }^{60} \mathrm{Fe}$ to ${ }^{26} \mathrm{Al}$ ratio obtained during this analysis is always between 0.10 and 0.24 whatever the map used to represent the ${ }^{26} \mathrm{Al}$ line emission. For the best tracers map, it is (in general) $\sim 0.18$ and agrees with those previously obtained by [13, 15].

\section{Annihilation radiation spectrum}

\subsection{The Bulge}

The annihilation line distribution has been already deeply studied [23, 17, 24]. In the present analysis, we use the additional information contained in the associated ortho-positronium continuum emission. This component is less easy to catch since it has to be disentangled from the diffuse 
continuum and the global point-source emission, which have comparable intensities between 300 and $400 \mathrm{keV}$, for the inner Galaxy $\left(|l|<30^{\circ},|b|<10^{\circ}\right)$. This makes it difficult to identify distinct spatial morphologies for the positronium components. It is thus assumed to be described with the same spatial distribution component than the $511 \mathrm{keV}$ narrow line.

\subsection{The disk}

The disk morphology has been shown, in the $511 \mathrm{keV}$ narrow line, to extend up to $\sim 200^{\circ}$ in longitude and $\sim 5-8^{\circ}$ in latitude but with limited information about its precise shape. In another hand, the diffuse emission (due to Inverse Compton of cosmic rays) has been explained by the superposition of an IC GALPROP and a $4.9 \mu$ DIRBE (much narrower in latitude) maps. Keeping in mind that the addition of different spatial morphologies in the sky model to describe two weak disk emissions would mainly generate "cross-talk" between these different sky components (mathematical degeneracy), we chose to describe the spatial distributions of the annihilation line and ortho-positronium continuum with the same ingredients (IC and 4.9 $\mu$ DIRBE maps), but with independent normalization factors.

\subsection{Results}

Both background method depicted in sec. 4.1 and 4.2 have been done with the annihilation radiation spectrum. The difference in the recovered fluxes with both methods, on the line and the ortho-positronium components, are less than $1 \%$ for the bulge and less than 5\% for the orthopositronium component. As main results, we find that, although slightly model-dependent, the positronium fraction is close to $100 \%$ and the bulge to disk (including halo for some models) flux ratio, for both the line and the positronium continuum is $\sim 0.2-0.3$.

It appears that the "disk" drawn by the annihilation radiation follows mainly the IC map. Since the GALPROP predicted map presents a large spheroid like structure in the inner parts (with $28 \%$ of the emission inside $|l, b|<30^{\circ}$ ), it gives a morphology very similar to that of the disk + halo model used in e.g. [23] or [17]. Note that we found the same integrated flux.

\section{Summary}

We found that our global diffuse spectrum is self-consistent through the measured characteristics of nuclear lines $\left({ }^{26} \mathrm{Al},{ }^{60} \mathrm{Fe}\right)$ and annihilation radiation spectrum. The results on the diffuse emission spectrum are presented elsewhere. The main point is that the IC emission distribution predicted by the GALPROP code is in fair agreement with the data, but a model with an electron spectrum increased by a factor 2 over the standard model based on the electrons (plus positrons) measured by Fermi-LAT still agrees better. In our preliminary analysis, the ${ }^{26} \mathrm{Al}$ line is detected with a $\sim 15 \sigma$ significance. The flux contained in the Inner Galaxy is around $2.7 \times 10^{-4} \mathrm{ph} \mathrm{cm}^{-2}$ $\mathrm{s}^{-1}$, in agreement with the value reported by [12]. It appears that the best templates to reproduce the ${ }^{26} \mathrm{Al}$ (and used also for the ${ }^{60} \mathrm{Fe}$ line) are the FIR maps with wavelengths 60 to $240 \mu$ or 53 $\mathrm{GHz}$ (free-free and dust) or to a lesser degree of confidence, NIR $4.9 \mu$ extinction-corrected maps for the high "contrast" maps. For low "contrast", $25 \mu, 12 \mu$ or IC maps provides also a good description of the line morphology in emission. Actually, our data does not allow us to distinguish between "low" and "high" contrast maps. From COMPTEL data, it had been concluded that the 
best tracers were DIRBE $240 \mu, 140 \mu, 100 \mu$ and $53 \mathrm{GHz}$ free-free maps. However, in this type of large-scale structures analysis, some systematics have not yet been clearly identified, and the instrumental response at wide angles remains very simplified. This limits again the weight of our conclusions. However, our results confirm that the best templates to described the ${ }^{26} \mathrm{Al}$ emission corresponds to those tracing the distribution of the extreme Population I; the most massive stars in the Galaxy [26]. The ${ }^{60} \mathrm{Fe}$ average flux agrees well with the value of $\sim 5 \times 10^{-5} \mathrm{ph} \mathrm{cm}^{-2} \mathrm{~s}^{-1}$ obtained by [13, 15]. The ratio between ${ }^{60} \mathrm{Fe}$ and ${ }^{26} \mathrm{Al}$ lines fluxes is around 0.18 , even if this value is not strongly constrained. (extreme values are 0.10 and 0.24 ).

As another topic and in complement to previous analysis based on the $511 \mathrm{keV}$ line emission, we have studied here the whole annihilation radiation, including the positronium continuum. The main result is that the characteristics of both the line and ortho-positronium continuum are quite homogeneous both in the bulge and the disk. However, these conclusion is based on the assumption that the ortho-positronium continuum follows the same spatial distribution than the $511 \mathrm{keV}$ line, since it is not possible to go further in refining the spatial geometry of the disk of the ortho-positronium continuum.

Finally, it is worth to mention that there is no detection in the SPI energy range of the 'Fermi bubbles'.

\section{References}

[1] Roques, J. P. et al., $A \& A$, 2003, 411, L91

[2] Vedrenne, G. et al., $A \& A$, 2003, 411, L63

[3] Bouchet, L., Strong, A., Porter, T.A, et al., 2011, ApJ , 739, 29

[4] Pls̈chke, S., Diehl, R., Schönfelder, V., Bloemen, H., et al., 2001, "Exploring the gamma-ray universe", Proceedings of the Fourth INTEGRAL Workshop, Editor: B. Battrick, Scientific editors: A. Gimenez, V. Reglero \& C. Winkler. ESA SP-459, Noordwijk: ESA Publications Division, ISBN 92-9092-677-5

[5] Bouchet L., Amestoy P. R.,Buttari A., et al., 2012, submitted to $A \& A$

[6] Porter, T. A., Moskalenko, I. V., Strong, A. W., et al., 2008, ApJ , 682, 400

[7] Abdo, A. A., et al., Phys. Rev. Lett., 2010, 104, 10, id. 10110

[8] Strong, A.W., 2010, Proceedings of the ICATPP Conference on Cosmic Rays for Particle and Astroparticle Physics, to be published by World Scientific (Singapore), arXiv:1101.1381.

[9] Krivonos, R., Revnivtsev, M., Churazov, E., et al., 2007, A\& A , 463, 957

[10] Bouchet, L., Jourdain, E., Roques, J. P, et al., 2008, ApJ , 679, 1315

[11] Türler, M., Chernyakova, M., Courvoisier, T. J.-L., et al., 2010, $A \& A$, 512, 49

[12] Wang, W., Lang, M. G., Diehl, R., et al., 2009, $A$ \& A , 496, 713

[13] Smith, D. M., 2004, ESA-SP-552, 45

[14] Harris, M. J, Knödlseder, J., Jean, P., et al., 2005, $A \& \&$, 433, L49

[15] Wang, W., Harris, M. J., Diehl, R., et al., 2007, $A \&$ \& , 469, 1005

[16] Bouchet, L., et al., 2012, in preparation

[17] Bouchet, L., Roques, J. P., \& Jourdain, E., ApJ , 2010, 720, 1772

[18] Diehl, R., Lang, M.G., Martin, P., Ohlendorf, H., et al., 2010, A \& A, 522, A51

[19] Martin, P., Knödlseder, J., Diehl, R. \& Meynet G., 2009 A \& A , 506, 703

[20] Voss, R., Martin, P., Diehl, R., Vink, J., Hartmann, D.H., et al., 2012, A \& A , arXiv:1202.028v1

[21] Timmes, F. X., Woosley, S. E., Hartmann, D. H., et al. 1995, ApJ , 449, 204

[22] Limongi, M., \& Chieffi, A. 2006, ApJ , 647, 483

[23] Weidenspointner, G., Skinner, G., Jean, P., et al., 2008, Nature, 451, 159

[24] Churazov, E., Sazonov, S., Ysygankov S., et al., 2011, MNRAS, 411(3), 1727

[25] Knödlseder, J., Bennett, K., Bloemen, H., et al. 1999, A \& A , 344, 68

[26] Diehl, R., Dupraz, C., Bennett, K., et al., 1995, $A \& A$, 298, 445 